Arq. Bras. Med. Vet. Zootec., v.67, n.6, p.1646-1652, 2015

\title{
Matrizes ovinas cruzadas são sempre mais eficientes do que as matrizes puras?
}

[Are sheep crosses always more efficient than pure sheep?]

\author{
G. Bianchi ${ }^{1}$, S. Carvalho ${ }^{2 *}$, J. Rivero ${ }^{1}$
}

\begin{abstract}
${ }^{1}$ Universidad de la República- Facultad de Agronomía- Estación Experimental "Dr. Mario A. Cassinoni”. Departamento de Producción Animal y Pasturas- Uruguay

${ }^{2}$ Universidade Federal de Santa Maria-UFSM- Santa Maria, RS. Bolsista de pós-doutorado do CNPq
\end{abstract}

\section{RESUMO}

Foi estudado o desempenho reprodutivo de 482 ovelhas multíparas (3/4 Texel x 1/4 Corriedale: TXC, 1/2 Romney Marsh x 1/2 Corriedale: RMC, 3/4 Poll Dorset x 1/4 Corriedale: PDC, 1/2 Finnish Landrace x 1/2 Merino Australiano: FLMA e Merino Australiano puras: MA), as características do crescimento de 585 cordeiros provenientes de carneiros Dorper e ovelhas: TXC $(\mathrm{n}=68)$; RMC $(\mathrm{n}=64)$; PDC $(\mathrm{n}=68)$; FLMA $(\mathrm{n}=183)$ e MA $(\mathrm{n}=202)$ e os quilogramas de carne de cordeiros produzidos/kg de ovelha colocadas em cobertura (como indicador de eficiência), pastoreando campo natural e pastagens cultivadas. As ovelhas FLMA e TXC apresentaram as maiores taxas de parição (167,89 e 174,36\%, respectivamente) em relação às demais cruzas, as quais não diferiram entre si (121,43 e 125,49\%, PDC e RMC, respectivamente; $\mathrm{P}>0,05)$, porém foram superiores as MA $(88,99 \%)$, sendo esse resultado uma consequência da maior quantidade de cordeiros nascidos/ovelha parida, que influenciou mais do que sua maior taxa de fertilidade. $O$ genótipo da ovelha também afetou $(\mathrm{P}<0,0001)$ o crescimento dos cordeiros até o momento da sua venda com 41,6 $\pm 6,7 \mathrm{~kg}$ aos $206 \pm 7,25$ dias de idade. Os cordeiros filhos das ovelhas TXC, PDC e RMC apresentaram maiores ganhos de peso diário que os filhos de FLMA, e esses maiores do que os MA. No entanto, de todas as cruzas avaliadas, somente a FLMA foi mais eficiente que a MA.

Palavras-chave: cruzamentos múltiplos, ovelhas cruzas Finnish Landrace, Merino Australiano, quilograma de cordeiro vendido/kg, ovelha encarneirada

\begin{abstract}
The reproductive performance of 482 multiparous ewes, with growth characteristics of 585 lambs from Dorper rams and ewes: $3 / 4$ Texel $x 1 / 4$ Corriedale (TXC, $n=68) ; 1 / 2$ Romney Marsh $x 1 / 2$ Corriedale $(R M C$, $n=64) ; 3 / 4$ Poll Dorset $x$ 1/4 Corriedale (PDC, $n=68) ; 1 / 2$ Finnish Landrace $x$ Australian Merino (FLAM, $n=183)$ and Australian Merino pure ewes $(A M, n=202)$ and $\mathrm{kg}$ of lamb produced/kg of sheep used in the service (as an indicator of efficiency), grazing natural and sown pastures, were studied._The TXC and FLAM sheep had the highest lambing percentage (167.89 and $174.36 \%$, respectively) compared to the rest of the other crosses, and did not differ from each other $(121.43$ and $125.49 \%, P D C$ and RMC respectively, $P>0.05)$, but were higher than $A M(88.99 \%)$, due to the greater number of lambs born / ewe calving rather than their higher fertility. The type of ewe also affected $(P<0.0001)$ lamb growth until its sale at $41.6 \pm 6.7 \mathrm{~kg}$ to $206 \pm 7.25$ days of age, showing mothers of TXC lambs, PDC and RMC had higher daily gains than the FLAM and these more than those of MA._However, in all crosses tested, only FLAM was more efficient than MA.
\end{abstract}

Keywords: multiple crosses, Finnish Landrace sheep crosses, Australian Merino, lamb sold kg/kg sheep served

Recebido em 12 de agosto de 2014

Aceito em 2 de junho de 2015

*Autor para correspondência (corresponding author)

E-mail: scarvalhoufsm@hotmail.com 


\section{INTRODUÇÃO}

A existência de heterose para as características produtivas e reprodutivas já se encontra suficientemente documentada em ovinos (Sidwell e Miller, 1971a; 1971b; 1971c; Nitter, 1978). O uso de cruzamentos múltiplos permite, além de explorar a heterose individual, aproveitar as diferenças raciais usando matrizes cruzadas e também fazer uso da heterose materna, em geral utilizada para as características de baixa herdabilidade e de ampla resposta, como as relacionadas à reprodução (Cardellino e Rovira, 1987).

Garibotto (1997) realizou uma exaustiva revisão sobre o aporte dos cruzamentos múltiplos na reprodução, produção de leite das ovelhas e, como consequência, sobre a velocidade de crescimento dos cordeiros. Esse autor verificou que não necessariamente o uso de matrizes cruzadas tem sempre que produzir resultados mais satisfatórios do que a alternativa de se usar a raça nativa e/ou tradicionalmente criada em determinada região. Bianchi e Garibotto (2007), ao trabalharem com ovelhas Milchschaf $x$ Corriedale, Ile de France x Corriedale, Texel x Corriedale e Corriedale puras, concluíram que, com exceção da cruza com Milchschaf, nenhuma outra superava o desempenho da Corriedale pura, contemplando, além de sua taxa reprodutiva e do crescimento dos cordeiros, sua produção de lã e maior consumo de qualquer das cruzas em comparação com a raça laneira pura.

Em termos comerciais, particularmente nos pequenos produtores rurais, que são a maioria em todo o Cone Sul e, também, os que menos terra e animais possuem, é muito comum a prática de reter as fêmeas oriundas de qualquer cruzamento, seja porque consideram que, pelo simples fato de serem cruzas, serão melhores matrizes ou, sobretudo, porque pretendem aumentar a quantidade de ventres para reproduzir, seja por ambas as razões. No entanto, não se encontram antecedentes que comparem simultaneamente o desempenho de matrizes cruzadas de raças de carne, prolíficas, em relação a matrizes laneiras.

O objetivo do presente trabalho foi avaliar o efeito de diferentes genótipos maternos sobre o seu desempenho reprodutivo, o crescimento de seus cordeiros, os quilogramas de cordeiros desmamados ou vendidos por ovelha parida, bem como determinar (como um indicador de eficiência de produção) os quilogramas de cordeiros desmamados ou vendidos por $\mathrm{kg}$ de ovelha colocada em reprodução.

\section{MATERIAL E MÉTODOS}

O trabalho foi desenvolvido no Setor de Ovinocultura da Estação Experimental "Dr. Mario A. Cassinoni”, da Faculdade de Agronomia, em Paysandú, Uruguai $\left(32,5^{\circ}\right.$ de latitude sul e $58,0^{\circ}$ de longitude oeste), no período compreendido entre a parição das ovelhas (06 de agosto a 16 de setembro de 2013) e o momento de venda dos cordeiros realizada em data fixa (08 de março de 2014).

Foram utilizadas 482 ovelhas multíparas (3/4 Texel x $1 / 4$ Corriedale: TXC, $n=39 ; 1 / 2$ Romney Marsh x 1/2 Corriedale: RMC, $\mathrm{n}=51 ; 3 / 4$ Poll Dorset $x$ 1/4 Corriedale: PDC, $n=56$; $1 / 2$ Finnish Landrace x 1/2 Merino Australiano: FLMA, $n=$ 109 e Merino Australiano puras: MA, $n=227$ ). As ovelhas foram sincronizadas utilizando-se duas doses de PGF2 $\alpha$ (com intervalo de sete dias entre si) e inseminadas com o sêmen de três carneiros da raça Dorper. A inseminação artificial foi realizada no período de 11 a 22 de março de 2013, e posteriormente se realizou o repasse com monta natural durante 18 dias adicionais, finalizando o serviço de cobertura em 8 de abril de 2013.

Foram utilizados também 585 cordeiros provenientes do cruzamento entre carneiros Dorper e as ovelhas: TXC $(\mathrm{n}=68)$; RMC $(\mathrm{n}=$ $64)$; PDC $(n=68)$; FLMA $(n=183)$ e MA $(n=$ 202).

As ovelhas permaneceram sobre pastagem cultivada de Trifolium repense Cichorium intibus, durante o período de cobertura, momento no qual se realizou a pesagem e a determinação do escore de condição corporal segundo Jefferies (1961), adaptado por Russel et al. (1969). Já durante as fases inicial e intermediária de gestação, as ovelhas permaneceram em pastagem natural, com distribuição de forragem primaveraverão: Desmodium incanum, Axonopus, Bromus aulecticus, Paspalum notatum, Setariageniculata e Stipa setigera e macegas de campo sujo: Eryngium horridum. A partir do terço final de gestação até o momento da parição, as ovelhas permaneceram em pastagem cultivada de 
Trifolium repens, Cichorium intibus e Lolium multiflorum.

Durante o período de parição, foram realizadas duas recorridas diárias de campo (oito horas e 16h) para identificação dos cordeiros e registro de: sexo, tipo de parto, peso vivo ao nascimento e, eventualmente, para assistência a alguma dificuldade de parto.

A assinalação foi realizada com uma idade e

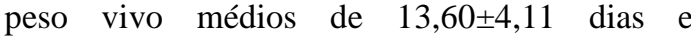
$9,33 \pm 2,44 \mathrm{~kg}$, respectivamente. Nesse momento, todos os cordeiros foram identificados com o sinal de propriedade em ambas as orelhas, e, em todos os machos, foi realizado o procedimento de criptorquidismo induzido (Hudson et al., 1968). Nesse momento, todos os cordeiros foram vacinados contra clostridioses e ectima contagioso. Administrou-se nas ovelhas um hantielmíntico à base de ivermectina $(1 \mathrm{~mL}$ para cada $50 \mathrm{~kg}$ de peso vivo).Trinta dias após a aplicação da primeira dose de vacina contra colostridioses, ocorreu a aplicação de uma segunda dose da vacina nos cordeiros.

Durante todo o período de lactação, as ovelhas e os seus respectivos cordeiros permaneceram como um único lote em pastagem cultivada de Trifolium repens, Cichorium intibus e Lolium multiflorum, com uma carga animal aproximada de oito a 10 ovelhas com seus cordeiros/ha.

O desmame dos cordeiros foi realizado com uma idade e peso vivo médios de 114,22 $\pm 7,24$ dias e $32,51 \pm 6,22 \mathrm{~kg}$, respectivamente. Para realizá-lo e evitar possíveis mastites nos úberes das ovelhas e também para favorecer o manejo posterior dos cordeiros, manteve-se a unidade ovelha-cria por $24 \mathrm{~h}$ junta, contida em um piquete, na sombra, sem acesso a água e alimento. Após esse período, os cordeiros foram separados das suas mães, e ambos foram mantidos encerrados por um período de $24 \mathrm{~h}$, durante o qual os cordeiros tinham acesso somente a água (ad libitum), e as ovelhas permaneciam sem água. Nesse momento, os cordeiros e as ovelhas foram dosificados com anti-helmíntico à base de closantel $\quad(10 \mathrm{mg} / \mathrm{kg})$. Posteriormente, os cordeiros retornaram à mesma pastagem utilizada durante o período de lactação, e as ovelhas permaneceram em uma área de campo com pastagem natural.
A partir do desmame, por um período de 10 dias, todos os cordeiros permaneceram sob a mesma condição de pastejo do período de lactação. Após esse período, passaram a pastejar, de forma restringida (das $19 \mathrm{~h}$ às sete horas do dia seguinte), um cultivo de soja (Glycine max), com uma carga animal de 30 cordeiros/ha, até sua comercialização, que ocorreu 92 dias após. Durante o dia, os cordeiros permaneceram encerrados, na sombra e com água ad libitum, recebendo como suplemento grão de sorgo inteiro na proporção de $1 \%$ do peso vivo.

Os cordeiros foram pesados periodicamente, com intervalos de 21 dias, até a sua comercialização. No momento que precedia o embarque para abate, os cordeiros foram pesados, após jejum de sólidos de 14 horas, e avaliados em relação ao escore de condição corporal.

O delineamento experimental utilizado foi $\mathrm{o}$ inteiramente ao acaso, com número desigual de repetições por tratamento. Para as avaliações de peso vivo, escore de condição corporal e para todas as variáveis reprodutivas (ovelhas falhadas = ovelhas vazias/ovelhas cobertas; taxa de repetição de cio $=$ ovelhas repetidas/ovelhas cobertas; fertilidade $=$ ovelhas paridas/ovelhas cobertas; prolificidade $=$ cordeiros nascidos/ovelhas paridas; parição $=$ cordeiros nascidos/ovelhas cobertas; tipo de parto $=$ partos simples, duplos ou triplos/partos totais; assinalação $=$ cordeiros assinalados/cordeiros nascidos; desmame $=$ cordeiros desmamados/cordeiros nascidos; mortalidade neonatal $=$ cordeiros mortos até 72 horas após a parição/cordeiros nascidos; mortalidade até o desmame $=$ total de cordeiros mortos entre o nascimento e o desmame/cordeiros nascidos), foi testado o efeito do genótipo da ovelha por meio de análise de variância e teste $\mathrm{F}$, adotando-se o nível de 5\% de significância.O modelo estatístico utilizado foi:

$Y_{i j}=\mu+G_{i}+\varepsilon_{i j}$,

em que:

$\mathrm{Y}_{\mathrm{ij}}=$ variáveis dependentes

$\mu=$ média geral das observações;

$\mathrm{G}_{\mathrm{i}}=$ efeito do genótipo da matriz $(\mathrm{i}=1 . . .5)$;

$\varepsilon_{\mathrm{ij}}=$ erro aleatório residual.

Para avaliação das variáveis relacionadas ao crescimento (peso vivo e ganho de peso diário) e escore de condição corporal dos cordeiros, foi testado o efeito do genótipo da ovelha, corrigido 
para sexo dos cordeiros e tipo de parto, por meio de análise de variância e teste $\mathrm{F}$, utilizando-se a idade dos cordeiros como covariável e adotandose o nível de 5\% de significância. O modelo estatístico utilizado foi:

$\mathrm{Y}_{\mathrm{ijklm}}=\mu+\mathrm{G}_{\mathrm{i}}+\mathrm{S}_{\mathrm{j}}+\mathrm{P}_{\mathrm{k}}+\mathrm{I}_{1}+\varepsilon_{\mathrm{ijklm}}$, em que:

$\mathrm{Y}_{\mathrm{ijklm}}=$ variáveis dependentes;

$\mu=$ média geral das observações;

$\mathrm{G}_{\mathrm{i}}=$ efeito do genótipo da matriz $(\mathrm{i}=1 . . .5)$;

$\mathrm{S}_{\mathrm{j}}=$ efeito do sexo do cordeiro $(\mathrm{j}=1$ a 2$)$;

$\mathrm{P}_{\mathrm{k}}=$ efeito do tipo de parto $(\mathrm{j}=1,2,3)$;

$\mathrm{I}_{1}=$ efeito da covariável idade do cordeiro;

$\varepsilon_{\mathrm{ijklm}}=$ erro aleatório residual.

Para calcular os quilogramas de cordeiros desmamados ou comercializados/ovelha parida, foram utilizados os dados de 374 fêmeas, incluindo-se no cálculo aquelas ovelhas em que ocorreu mortalidade de cordeiros em ambas as fases de avaliação: do nascimento ao desmame e entre o desmame e a comercialização. Adicionalmente, e assumindo-se que o consumo por unidade de peso é constante (Oficialdegui, 1992), considerou-se o cociente $\mathrm{kg}$ de cordeiro produzido na venda $/ \mathrm{kg}$ de ovelha na cobertura, como uma estimativa de eficiência para ambos os genótipos. Para o contraste de médias, foi utilizado o teste " $\mathrm{t}$ " de Student, adotando-se o nível de 5\% de significância. Para realização das análises, foi utilizado o procedimento GLM do pacote estatístico SAS, versão 9.1 (SAS, 2004).

\section{RESULTADOS E DISCUSSÃO}

Na Tab. 1, são apresentados os indicadores do desempenho reprodutivo dos diferentes genótipos maternos, assim como o peso vivo e o escore de condição corporal das ovelhas no início do período de cobertura.

As ovelhas cruzas TXC, RMC e PDC apresentaram peso vivo e, sobretudo, estado corporal dentro da faixa considerada adequada para o período de cobertura (Ruseel et al., 1969). No entanto, em relação às cruzadas FLMA e as MA, embora elas tenham apresentado um peso satisfatório para ambos os genótipos (Bianchi et al., 2013), seu estado corporal se encontrava abaixo do mínimo desejável para um bom desempenho na parição (ECC $\geq 3$; Ruseel et al., 1969), particularmente no caso das ovelhas puras. Essa situação explica a maior quantidade de fêmeas falhadas e, como consequência, a menor fertilidade do genótipo MA, particularmente em relação às cruzas TXC e FLMA, que foram as que apresentaram maior taxa de fertilidade.

Tabela 1. Valores médios e erro-padrão para peso vivo, escore de condição corporal e desempenho reprodutivo de ovelhas multíparas, cruzadas e puras

\begin{tabular}{|c|c|c|c|c|c|c|}
\hline \multirow[b]{2}{*}{ Variáveis } & \multicolumn{5}{|c|}{ Genótipos } & \multirow[b]{2}{*}{ Prob. } \\
\hline & TXC & RMC & $\mathrm{PDC}$ & FLMA & MA & \\
\hline Peso vivo (kg) & $70,17 \pm 1,38 \mathrm{a}$ & $62,17 \pm 1,24 b$ & $70,21 \pm 1,18 \mathrm{a}$ & $55,04 \pm 0,80 \mathrm{c}$ & $42,68 \pm 0,69 d$ & $* * *$ \\
\hline Repetição de cio (\%) & $10,26 \pm 4,67$ & $7,84 \pm 4,08$ & $12,50 \pm 3,89$ & $5,50 \pm 2,79$ & $10,57 \pm 1,93$ & Ns \\
\hline Fertilidade $(\%)$ & $97,44 \pm 5,97 \mathrm{a}$ & $80,39 \pm 5,22 b c$ & $85,71 \pm 4,98 \mathrm{abc}$ & $91,74 \pm 3,57 \mathrm{ab}$ & $75,33 \pm 2,47 \mathrm{c}$ & $* * *$ \\
\hline Ovelhas falhadas (\%) & $2,56 \pm 5,97 \mathrm{c}$ & $19,61 \pm 5,22 \mathrm{ab}$ & $14,29 \pm 4,98 \mathrm{abc}$ & $8,26 \pm 3,57 b c$ & $24,67 \pm 2,47 a$ & $* * *$ \\
\hline Prolificidade (\%) & $179 \pm 10 \mathrm{ab}$ & $156 \pm 10 b c$ & $142 \pm 8 c$ & $183 \pm 5 \mathrm{a}$ & $118 \pm 4 d$ & $* * *$ \\
\hline Partos simples (\%) & $31,58 \pm 7,20 \mathrm{c}$ & $46,34 \pm 6,94 b c$ & $64,58 \pm 6,41 b$ & $35,00 \pm 4,44 \mathrm{c}$ & $81,87 \pm 3,40 \mathrm{a}$ & $* * *$ \\
\hline Partos duplos (\%) & $57,89 \pm 7,31 \mathrm{a}$ & $51,22 \pm 7,03 \mathrm{a}$ & $29,17 \pm 6,50 b$ & $47,00 \pm 4,50 \mathrm{a}$ & $18,13 \pm 3,44 b$ & $* * *$ \\
\hline Partos triplos (\%) & $10,53 \pm 3,85 \mathrm{ab}$ & $2,44 \pm 3,71 b c$ & $6,25 \pm 3,42 b c$ & $18,00 \pm 2,37 \mathrm{a}$ & $0,00 \mathrm{c}$ & $* * *$ \\
\hline Assinalação (\%) & $164,10 \pm 11,35 \mathrm{a}$ & $121,57 \pm 9,93 b$ & $112,50 \pm 9,48 b$ & $155,96 \pm 6,79 a$ & $79,29 \pm 4,71 \mathrm{c}$ & $* * *$ \\
\hline
\end{tabular}

${ }^{1}$ Mortalidade neonatal (até 72 horas após o parto).

${ }^{2}$ Mortalidade do nascimento ao desmame.

Ns: $(\mathrm{P}>0,05)$; $:(\mathrm{P} \leq 0,05)$. ***: $(\mathrm{P} \leq 0,001)$. Médias, na mesma coluna, seguidas de letras distintas, diferem entre si $(\mathrm{P} \leq 0,05)$. 
Tabela 2. Valores médios e erro-padrão para os pesos vivos, ganhos de peso diários durante o período experimental e o escore de condição corporal no momento da comercialização dos cordeiros

\begin{tabular}{lcccccc}
\hline \multirow{2}{*}{$\begin{array}{c}\text { Genótipos } \\
\text { maternos }\end{array}$} & $\begin{array}{c}\text { PVN } \\
(\mathrm{kg})\end{array}$ & $\begin{array}{c}\text { PVD } \\
(\mathrm{kg})\end{array}$ & $\begin{array}{c}\text { PVC } \\
(\mathrm{kg})\end{array}$ & $\begin{array}{c}\text { GMDND } \\
(\mathrm{g} / \mathrm{d})\end{array}$ & $\begin{array}{c}\text { GMDDC } \\
(\mathrm{g} / \mathrm{d})\end{array}$ & $\begin{array}{c}\text { ECC } \\
(0-5)\end{array}$ \\
\cline { 2 - 7 } & $* * *$ & $* * *$ & $* * *$ & $* * *$ & $* * *$ & $* * *$ \\
\hline TXC & $4,40 \pm 0,09 \mathrm{a}$ & $33,35 \pm 0,62 \mathrm{ab}$ & $43,99 \pm 0,74 \mathrm{a}$ & $255 \pm 0,05 \mathrm{ab}$ & $192 \pm 0,03 \mathrm{a}$ & $3,87 \pm 0,27 \mathrm{a}$ \\
RMC & $4,01 \pm 0,10 \mathrm{~b}$ & $32,46 \pm 0,64 \mathrm{bc}$ & $41,92 \pm 0,78 \mathrm{~b}$ & $250 \pm 0,05 \mathrm{bc}$ & $184 \pm 0,04 \mathrm{ab}$ & $3,69 \pm 0,29 \mathrm{bc}$ \\
PDC & $4,32 \pm 0,09 \mathrm{a}$ & $34,98 \pm 0,61 \mathrm{a}$ & $44,10 \pm 0,71 \mathrm{a}$ & $267 \pm 0,05 \mathrm{a}$ & $192 \pm 0,03 \mathrm{a}$ & $3,78 \pm 0,26 \mathrm{ab}$ \\
FLMA & $3,72 \pm 0,06 \mathrm{c}$ & $31,42 \pm 0,42 \mathrm{c}$ & $40,50 \pm 0,47 \mathrm{~b}$ & $240 \pm 0,03 \mathrm{c}$ & $178 \pm 0,02 \mathrm{~b}$ & $3,72 \pm 0,18 \mathrm{~b}$ \\
MA & $3,85 \pm 0,06 \mathrm{bc}$ & $27,43 \pm 0,49 \mathrm{~d}$ & $36,79 \pm 0,56 \mathrm{c}$ & $208 \pm 0,04 \mathrm{~d}$ & $159 \pm 0,03 \mathrm{c}$ & $3,62 \pm 0,21 \mathrm{c}$ \\
\hline
\end{tabular}

PVN = peso vivo ao nascimento; PVD = peso vivo ao desmame; PVC = peso vivo no momento da comercialização; GMDND = ganho de peso médio diário do nascimento ao desmame; GMDDC $=$ ganho de peso médio diário do desmame até a comercialização; ECC = escore de condição corporal no momento da comercialização.

***: $(\mathrm{P} \leq 0,001)$. Médias, na mesma coluna, seguidas de letras distintas, diferem entre si $(\mathrm{P} \leq 0,05)$.

$\mathrm{Na} \quad$ Tab. 3, estão apresentados os resultados referentes ao efeito dos diferentes genótipos maternos sobre as variáveis que contemplam o resultado conjunto do desempenho reprodutivo mais o crescimento dos cordeiros: $\mathrm{kg}$ de cordeiros desmamados ou comercializados/ovelha parida e, como indicador de eficiência de produção, a relação entre os quilogramas de peso vivo de cordeiros desmamados ou vendidos $/ \mathrm{kg}$ de ovelha colocada em cobertura.

As ovelhas cruzadas produziram mais quilograma de cordeiros ao desmame e no momento da venda em comparação a suas contemporâneas puras MA, particularmente aquelas FLMA, as quais, apesar de superarem somente as matrizes puras quanto ao crescimento dos seus cordeiros, foram as que apresentaram a maior taxa de prolificidade, corroborando a importância da reprodução na produção de carne. Contudo, as fêmeas TXC, RMC e PDC não apresentaram diferença em relação à cruza FLMA, sendo esse resultado proporcionado pelo seu bom desempenho reprodutivo e pela maior taxa de crescimento dos seus cordeiros.

Tabela 3. Valores médios e erro-padrão para os quilogramas de cordeiros desmamados ou comercializados por ovelha parida ou por quilograma de ovelha colocada em cobertura

\begin{tabular}{lcccc}
$\begin{array}{c}\text { Genótipos } \\
\text { maternos }\end{array}$ & $\begin{array}{c}\mathrm{KgCD} / \mathrm{OP} \\
(\mathrm{kg})\end{array}$ & $\begin{array}{c}\mathrm{KgCC} / \mathrm{OP} \\
(\mathrm{kg})\end{array}$ & $\begin{array}{c}\mathrm{KgCD} / \mathrm{kgOC} \\
(\mathrm{kg} / \mathrm{kg})\end{array}$ & $\begin{array}{c}\mathrm{KgCC} / \mathrm{kgOC} \\
(\mathrm{kg} / \mathrm{kg})\end{array}$ \\
\cline { 2 - 5 } & $* * *$ & $* * *$ & $* * *$ & $* * *$ \\
\hline TXC & $55,69 \pm 2,71 \mathrm{a}$ & $69,94 \pm 3,72 \mathrm{a}$ & $0,81 \pm 0,06 \mathrm{~b}$ & $1,01 \pm 0,08 \mathrm{~b}$ \\
RMC & $49,71 \pm 2,61 \mathrm{a}$ & $62,98 \pm 3,62 \mathrm{ab}$ & $0,82 \pm 0,06 \mathrm{ab}$ & $1,03 \pm 0,08 \mathrm{ab}$ \\
PDC & $48,59 \pm 2,41 \mathrm{a}$ & $59,15 \pm 3,27 \mathrm{~b}$ & $0,68 \pm 0,05 \mathrm{bc}$ & $0,84 \pm 0,07 \mathrm{~b}$ \\
FLMA & $51,29 \pm 1,91 \mathrm{a}$ & $64,46 \pm 2,63 \mathrm{ab}$ & $0,96 \pm 0,04 \mathrm{a}$ & $1,20 \pm 0,06 \mathrm{a}$ \\
MA & $28,85 \pm 1,36 \mathrm{~b}$ & $38,92 \pm 1,74 \mathrm{c}$ & $0,67 \pm 0,03 \mathrm{c}$ & $0,90 \pm 0,04 \mathrm{~b}$ \\
\hline
\end{tabular}

$\mathrm{KgCD} / \mathrm{OP}=\mathrm{kg}$ de cordeiros desmamados por ovelha parida; $\mathrm{KgCC} / \mathrm{OP}=\mathrm{kg}$ de cordeiros comercializados por ovelha parida; $\mathrm{KgCD} / \mathrm{kgOC}=\mathrm{kg}$ de cordeiros desmamados por $\mathrm{kg}$ de ovelha colocada em cobertura; $\mathrm{kgCC} / \mathrm{kgOC}=\mathrm{kg} \mathrm{de}$ cordeiros comercializados por $\mathrm{kg}$ de ovelha colocada em cobertura.

***: $(\mathrm{P} \leq 0,001)$. Médias, na mesma coluna, seguidas de letras distintas, diferem entre si $(\mathrm{P} \leq 0,05)$.

No entanto, tão importante como a renda obtida por ovelha encontram-se os custos e, dentro desses, os da alimentação. Ao serem as ovelhas TXC e PDC mais pesadas do que as FLMA, essas duas cruzas com raças de carne foram menos eficientes quanto à produção de cordeiros, sem diferenciarem-se das ovelhas puras MA. Se for considerada a produção de lã dos genótipos maternos FLMA e MA, particularmente quanto aos benefícios de terem um velo mais fino e de maior valorização (Bianchi et al., 2013), surgem diferenças com o genótipo RMC, que são ampliadas com o restante dos genótipos avaliados no presente trabalho. 


\section{CONCLUSÕES}

Conclui-se que nem sempre as matrizes cruzadas são mais eficientes do que as puras. Nesse sentido e apesar de os cordeiros filhos de ovelhas cruzas de raças de carne (TXC, RMC e PDC) apresentarem maior crescimento do que aqueles filhos de ovelhas FLMA (e esses mais que os puros), o genótipo materno que apresentou o melhor resultado comercial foi o FLMA.

\section{REFERÊNCIAS}

AZZARINI, M.; OFICIALDEGUI, R.; CARDELLINO, R. Sistemas alternativos de producción ovina: potenciación de la producción de carne en sistemas laneros. Prod. Ovina, v.9, p.7-20, 1996.

BAKER, R.L. Finnsheep and their utilizationexperiences in temperature conditions. J. Agric. Sci. Fin., v.60, p.455, 1988.

BIANCHI, G.; GARIBOTTO, G. Uso de madres híbridas y su impacto en el sistema de producción. In: BIANCHI G. (Ed). Alternativas tecnológicas para la producción de carne ovina de calidad en sistemas pastoriles. Montevideo: Hemisferio Sur, 2007. p.107-131.

BIANCHI, G.; GARIBOTTO, G.; BENTANCUR, O.; MENCHACA, A. Evaluación de la Raza Finnish Landrace en sistemas estratificados de producción de carne, utilizando ovejas Merino Australiano y carneros Poll Dorset. Montevideo: Hemisfério Sur, 2013. 40p. (Serie FPTA-INIA, n.52).

BIANCHI, G.; GARIBOTTO, G.; LAMARCA, M.. Estudio comparativo de hembras Finnish Landrace $\mathrm{x}$ Merino Australiano vs. Merino Australiano: 1. Desempeño reproductivo. Abanico Vet., v.4, p.32-37, 2014.

BITTANTE, G.; GALLO, L.; CARNIER, P. et al. Effects on fertility and litter traits under accelerated lambing scheme in crossbreeding between Finnsheep and an Alpine sheep breed. Small Ruminant Res., v.23, p.43-50, 1996.

CARDELlINO, R.A.; ROVIRA, J. Mejoramiento genético animal. Montevideo: Hemisferio Sur, 1987. 253p.
CASAS, E.; FREKING, B.; LEYMASTER, K. Evaluation of Dorset, Finnsheep, Romanov, Texel and Montadale breeds of sheep: II. Reproduction of F1 ewes in fall mating seasons. J. Anim. Sci., v.82, p.1280-1289, 2004.

CLARKE, L.; YAKUBU, D.P.; SYMONDS, M.E. Influence of maternal bodyweight on size, conformation and survival of newborn lambs. Reprod. Fertil. Develop. v.9, p.509-514, 1997.

DUTRA F. Nuevos enfoques sobre la patología de la mortalidad perinatal de corderos. Montevideo: Hemisfério Sur, 2005. P.137-141. (Serie Técnica INIA, n.401).

FAHMY, M. The accumulative effect of Finnsheep breeding in crossbreeding schemes: market lamb production from crossbreed ewes. $J$. Anim. Sci., v.69, p.47-55, 1989.

FERNÁNDEZ ABELLA, D. Mortalidad neonatal de corderos. In: TEMAS de reproducción ovina e inseminación artificial en ovinos y bovinos. Montevideo: Facultad de Agronomia, 1995. p.39-60.

FOGARTY, N.; HOPKINS D; HOLST, P. Lamb production from diverse genotypes. Cowra Agriculture Research and Advisory Station: NSW Agriculture, 1998. 33p.

GANZABAL, A.; CIAPPESONI, G.; BANCHERO, G. et al. Biotipos maternales y terminales para enfrentar los nuevos desafíos de la producción ovina moderna. Montevideo: Hemisfério Sur, 2012. p.14-18. (Serie Técnica INIA, n.29).

GARIBOTTO, G. Desempeño productivo y reproductivo de madres y corderos cruza: Resultados de la Investigación Extranjera. In: BIANCHI G. (Ed). Producción de carne ovina en base a cruzamientos. Montevideo: Facultad de Agronomia, 1997. p.23-42.

GREEFF, J.C.; BOUWER, L.; HOFMEYR, J.H. Biological efficiency of meat and wool production of seven sheep genotypes. Anim. Sci., v.61, p.259-264, 1995.

HUDSON, L.W.; GIMP, H.A.; WOOLFOLK, P.G. et al. Effect of induced cryptorchidism at different weights on performance and carcass traits of lambs. J. Anim. Sci., v.27, p.45-47. 1968. 
JEFFERIES, B.J. Body condition scoring and its use in management. Tasm. J. Agric., v.32, p.19$21,1961$.

LIRA, F.R. Producción ovina mejorada en la zona austral de Chile. Punta Arenas: Instituto de Investigaciones Agropecuárias, 2007. 17p. (Boletín INIA, n.164).

MAZZITELLI, F. Algunas consideraciones sobre crecimiento de corderos. Bol. Técnico SUL, n.8, p.53-61, 1983.

MILNE C. The history of the Dorper sheep. Small Ruminant. Res., v.36, p.99-102, 2000.

NITTER, G. Breed utilisation for meat production in sheep. Anim. Breed. Abst., v.46, p.131-143, 1978.

OFICIALDEGUI, R. Factores que afectan el consumo y la eficiencia de los ovinos. In: SEMINARIO SOBRE MEJORAMIENTO GENÉTICO EN LANARES, 2., 1992, Piriápolis. Trabajos... Montevideo: Secretariado Uruguaio de Lana, 1992, p.167-183.
RUSSEL, A.J.F.; DONEY, J.M.; GUNN, R.G. Subjective assessment of body fat in live sheep. J. Agric. Sci. Camb., v.72, p.451-454, 1969.

SAS User's Guide.Version 9. North Caroline, SAS Institute INC., 2004. 5135p.

SIDWELL, G.M.; MILLER, L.R. Production in some pure breeds of sheep and their crosses. I. Reproductive efficiency in ewes. J. Anim. Sci., v.32, p.1084-1089, 1971a.

SIDWELL, G.M.; MILLER, L.R. Production in some pure breeds of sheep and their crosses. II. Birth weights and weaning weights of lambs. $J$. Anim. Sci., v.32, p.1090-1094, 1971 b.

SIDWELL, G.M.; MILLER, L.R. Production in some pure breeds of sheep and their crosses. III. Production indexes in ewes. J. Anim. Sci., v.32, p.1095-1098, 1971c. 